\title{
The $\lambda$ Bootis stars
}

\section{Ernst Paunzen}

Institute for Astronomy, University Vienna, Türkenschanzstr. 17, A-1180 Vienna, Austria email: Ernst.Paunzen@univie.ac.at

\begin{abstract}
A comprehensive overview of the $\lambda$ Bootis stars is presented. This small group (only a maximum of $2 \%$ of all objects in the relevant spectral domain) are Population I late B to early F-type stars, with moderate to extreme (up to a factor 100) surface underabundances of most Fe-peak elements and solar abundances of lighter elements (C, N, O, and S). They form a separate group among the classical chemically peculiar objects of the upper Main Sequence as their underabundances are quite outstanding. The basic membership criteria as well as the theories to explain the $\lambda$ Bootis phenomenon are covered. New observations including detailed elemental (surface) abundances, tests for pulsational instability, fluxes in the IR region, and measurements from the Hipparcos satellite permit one to make a statistical sound analysis of the group properties. Details of the evolutionary status, abundance pattern and tests for developed theories are presented.
\end{abstract}

Keywords. Stars: abundances, chemically peculiar, fundamental parameters, oscillations

\section{Introduction}

$\lambda$ Bootis stars are Population I late B to early F-type stars, with moderate to extreme (up to a factor 100) surface underabundances of most Fe-peak elements and solar abundances of lighter elements (C, N, O, and S). Only a maximum of about $2 \%$ of all objects (about 50 stars in total) in the relevant spectral domain are believed to be $\lambda$ Bootis type stars as referenced by Paunzen et al. (2001) and Gray \& Corbally (2002). Although the number of members is small, the group is an excellent test field for various astrophysical theories such as accretion, diffusion, mass-loss and asteroseismology.

\section{Classification resolution spectroscopy and membership criteria}

The following definition is primarily from Gray (1988). It summarizes the basic features of moderate dispersion spectra which seem to be shared by all well established $\lambda$ Bootis stars:

- The $\lambda$ Bootis stars are early-A to early-F type stars with an approximate spectral type range (based on the hydrogen lines) of B9.5 to F0 with possible members as late as F3.

- The $\lambda$ Bootis stars are characterized by weak Mg II $\lambda 4481$ lines, such that the ratio Mg II $\lambda 4481 /$ Fe I $\lambda 4383$ is significantly smaller than in normal stars. In addition the spectra exhibit a general metal-weakness. The typical shell lines such as, Fe II $\lambda 4233$, tend to be weak as well. But the $\lambda$ Bootis stars do not show the typical shell spectral characteristics.

- The following classes of stars should be excluded from the $\lambda$ Bootis group even if they show weak $\lambda 4481$ lines: shell stars, protoshell stars, He-weak stars (easily distinguished on the basis of their hydrogen line temperature types), and other CP stars. FHB and intermediate Population II stars may be distinguished from the $\lambda$ Bootis stars on the basis of their hydrogen line profiles. High- $v \sin i$ stars should be considered as $\lambda$ Bootis 
candidates only if the weakening of $\lambda 4481$ is obvious with respect to the standards with high values of $v \sin i$.

- $\lambda$ Bootis stars are also characterized by broad hydrogen lines, and in many cases, these hydrogen lines are exceptionally broad. In the late- $\mathrm{A}$ and early-F $\lambda$ Bootis stars, the hydrogen line profiles are often peculiar, and are characterized by broad wings, but shallow cores.

- The distribution of rotational velocities of the $\lambda$ Bootis stars cannot be distinguished from that of the normal Population I A-type stars.

This definition already includes the basic criteria for a successful classification of a true $\lambda$ Bootis star. A highly underestimated effect on the classification process is the influence of fast rotation on the derived spectral type. Slettebak et al. (1980) have shown that the equivalent width of $\lambda 4481$ decreases with increasing rotation for models later than A0 (see also, Abt \& Morrell 1995). A much stronger effect was found for $\mathrm{H} \gamma$. A fast rotating star will be classified much later using $\mathrm{H} \gamma$ than using $\mathrm{Mg}$ II $\lambda 4481$. One would classify a rapidly rotating true $\mathrm{A} 5$ type star as $\mathrm{hF} 1 \mathrm{~mA} 7$ or metal-weak.

Paunzen (2001) performed an extensive classification resolution spectroscopic survey in the Galactic field and nine open clusters to detect new $\lambda$ Bootis stars. He confirmed the membership of 18 candidates and detected 26 new $\lambda$ Bootis stars. The survery by Gray \& Corbally (2002) was devoted to open clusters. They noticed the apparent absence of $\lambda$ Bootis stars in 24 open clusters with ages from $15 \mathrm{Myr}$ to $700 \mathrm{Myr}$.

The list of members has steadily evolved since the compilation of Gray (1988) to that of Paunzen et al. (2002a). This evolution is purely based on observations which are fully documented in each reference. An uncritical evaluation of members as used in Gerbaldi et al. (2003) is not appropriate for drawing statistical conclusions about the incidence of undetected spectroscopic binary systems or the heterogeneity of this group.

\section{The abundance pattern of $\lambda$ Bootis stars.}

Only recently have abundances become available for about half of all $\lambda$ Bootis stars. Heiter (2002) draw conclusions about the abundance pattern of the group, investigated their statistical properties and compared the abundances with the interstellar medium. In a few cases, the abundances differ by more than 0.6 dex and these have been discarded. Abundances for spectroscopic binaries which have been treated as single stars have not been taken into account. The following conclusions were made:

- The abundances of $\mathrm{C}, \mathrm{N}$ and $\mathrm{O}$ are on the average solar, but considerable underand overabundances $(-0.8$ and +0.6 dex $)$ occur as well.

- The star-to-star scatter for the element abundances of C, N, O and S is smaller than for most of the heavier elements.

- The mean abundance of $\mathrm{Na}$ is also solar, but the star-to-star scatter is \pm 1 dex.

- The iron peak elements are more underabundant than C, N, O, and S for each star.

- The iron peak elements from Sc to Fe as well as Mg, Si, Ca, Zn, Sr and Ba are depleted by about -1 dex relative to the solar chemical composition.

- $\mathrm{Al}$ is slightly more depleted (-1.5 dex) and Ni, Y and Zr are slightly less depleted.

- The star-to-star scatter is twice as large as for a comparable sample of normal stars.

The results suggest the existence of a separate chemically peculiar group of " $\lambda$ Bootis stars" with a characteristic abundance pattern. The scatter within the abundance pattern is, for example, comparable to that of Am/Fm stars. Furthermore, the comparison to normal stars is difficult because the sample of "normal" Main Sequence stars with known abundances and parameters similar to that of the $\lambda$ Bootis stars is rather limited. 
Another way of characterizing the chemical composition of $\lambda$ Bootis stars is to examine abundance ratios between different elements. The most important finding was an anticorrelation between the ratios of light elements (C, N, O and $\mathrm{S}$ ) to heavier elements and the heavy element abundances. $[\mathrm{C} / \mathrm{Fe}] \gg 0$ holds for all $\lambda$ Bootis stars and the iron abundances are significantly lower than those found for superficially normal stars. On the other hand, there is an overlap with normal stars for other heavier elements found by Paunzen et al. (2002a).

\section{The characteristics of $\lambda$ Bootis stars in the UV region}

To summarize previous efforts, Faraggiana et al. (1990) tried to establish criteria for the definition of a homogeneous group of $\lambda$ Bootis stars.

Solano \& Paunzen $(1998,1999)$ presented careful analyses of the IUE final archive using the low and high resolution spectra separately. Their goal was to establish criteria in the UV region without using data from the optical domain and to unambiguously distinguish $\lambda$ Bootis from FHB stars.

From the low resolution spectra they concluded that a star is considered to be a member of the $\lambda$ Bootis group if:

- C I $\lambda 1657 / \mathrm{Al}$ II $\lambda 1670>2.0$

- C I $\lambda 1657 / \mathrm{Ni}$ II $\lambda 1741>2.5$

These limits have been set so that they are valid for the entire range of effective temperatures. Small uncertainties due to variations in $\log g$ and $[\mathrm{M} / \mathrm{H}]$ have also been taken into account to make these limits independent of physical parameters such as the effective temperature and the surface gravity. Moreover, they are also independent of the evolutionary status since a clear distinction between $\lambda$ Bootis and FHB stars was established. From the analysis of the high resolution spectra they were able to add the following criteria for a star being a member of the $\lambda$ Bootis group:

- C I $\lambda 1657 / \mathrm{Si}$ II $\lambda 1527>3.0$

- C I $\lambda 1657 /$ Ca II $\lambda 1839>8.0$

These four unambiguous criteria in the UV region reflect the typical abundance pattern for this group (Section 3).

\section{The characteristics of $\lambda$ Bootis stars in the IR region}

King (1994) and Fajardo-Acosta et al. (1999) gave brief summaries of some observations available in the IR. From these references it was already clear that 1) only very few $\lambda$ Bootis members were detected in the IR and 2) even fewer objects show infrared excesses.

Paunzen et al. (2003) presented all available data for members of the $\lambda$ Bootis group in the wavelength region beyond $7000 \AA$. The data include spectrophotometry, Johnson RIJHKLM and 13-color photometry as well as IRAS and ISO measurements. The data were homogeneously reduced and transformed into a standard system given in units of Jansky. In total, measurements for 34 (26 with data redward of $20 \mu \mathrm{m}$ ) well established $\lambda$ Bootis stars are available. From those 26 objects, 6 show an infrared excess and 2 doubtful detections resulting in $23 \%$ showing signs of circumstellar or interstellar material around them.

For four stars the infrared excess was detected at two different wavelengths which allowed one to fit the data by a simple model. The derived dust temperatures are between 70 and $200 \mathrm{~K}$ and the fractional dust luminosities range from $2.2 \times 10^{-5}$ to $4.3 \times 10^{-4}$. These values are comparable with those found for Vega-type objects. 
ISO-SWS spectroscopy for HD 125162 and HD 192640 resulted in the detection of pure stellar $\mathrm{H}$ I lines ruling out an active accretion disk around these objects. Furthermore, a search for the $\mathrm{CO}(2-1)$ line at $238.538 \mathrm{GHz}$ and continuum observations at $347 \mathrm{GHz}$ of three $\lambda$ Bootis stars yield only upper limits.

\section{Pulsational characteristics}

In general, chemical peculiarities found for stars on the Upper Main sequence exclude $\delta$ Scuti type pulsation (e.g., Ap and Am stars), but for the $\lambda$ Bootis stars it is just the opposite which makes them very interesting for asteroseismological investigations.

Paunzen et al. (2002b) summarize the overall pulsational characteristics of the group members (with only two so far not photometrically investigated). They have analyzed the pulsational stability of the individual objects with typical upper limits for bona-fide "constant" objects of about $2 \mathrm{mmag}$ using also the photometric data of the Hipparcos satellite.

From all well established members (not all within the classical instability strip), 33 have been found to show typical $\delta$ Scuti type pulsation. With the already published abundance values, they have also searched for the existence of a Period-Luminosity-Color-Metallicty relation. They compared the characteristics of the $\lambda$ Bootis group to a sample of "normal" $\delta$ Scuti pulsators. The latter was chosen such that it matches the $\lambda$ Bootis stars within the global astrophysical parameters. The following properties of the $\lambda$ Bootis stars are different from those of the $\delta$ Scuti pulsators:

- At least $70 \%$ of all $\lambda$ Bootis type stars inside the classical instability strip pulsate (this number for "normal" stars is much less)

- Only a maximum of two stars may pulsate in the fundamental mode but there is a high percentage with $Q<0.020 \mathrm{~d}$ (high overtone modes). For $\delta$ Scuti stars this is just the opposite.

- The instability strip of the $\lambda$ Bootis stars at the ZAMS is 25 mmag bluer in $(b-y)_{0}$ than that of the $\delta$ Scuti stars.

No clear evidence for a [Z] correlation with period, luminosity or color was found. The Period-Luminosity-Color relation is the same as for the classical $\delta$ Scuti stars.

Bohlender et al. (1999) performed high resolution, high signal-to-noise spectroscopy (spectra centered at NaD $\lambda 5892$ and/or $\lambda 4500$ ) of $\lambda$ Bootis stars to investigate the incidence of high-degree nonradial pulsation. The discovered spectrum variability is very similar to that seen in rapidly rotating $\delta$ Scuti stars. For all but one of the investigated pulsators, high-degree nonradial modes were detected spectroscopically.

Photometric as well as spectroscopic variability is a common phenomenon in $\lambda$ Bootis stars. Several $\lambda$ Bootis stars are therefore excellent candidates for multisite photometric and spectroscopic campaigns or space based observations (e.g., MOST and COROT) to put further constraints on stellar interior and pulsation models.

Up to now, five $\lambda$ Bootis stars were targets of multisite campaigns: HD 15165, HD 105759, HD 111786, HD 142994 and HD 192640, see Paunzen et al. (2002b) for more details. For none of these objects a mode identification was possible up to now.

\section{The evolutionary status of the group}

Paunzen et al. (2002a) have used all currently available photometric data as well as Hipparcos data to determine astrophysical parameters such as the effective temperatures, surface gravities and luminosities. Masses and ages were calibrated within appropriate post-MS evolutionary models. Furthermore, Galactic space motions were calculated with 
the help of radial velocities from the literature. All results were compared with those of a test sample of normal-type objects in the same spectral range chosen to match the $(B-V)_{0}$ distribution of the $\lambda$ Bootis group. From a comprehensive statistical analysis they conclude:

- The standard photometric calibrations within the Johnson $U B V$, Strömgren uvby $\beta$ and Geneva 7-colour systems are valid for this group of chemically peculiar stars.

- The group of $\lambda$ Bootis stars consists of true Population I objects which can be found over the whole area of the MS with a peak at a rather evolved stage $(\approx 1 \mathrm{Gyr})$. That is in line with the distribution of the test sample.

- The $\lambda$ Bootis type group is not significantly distinct from normal stars except, possibly, by having slightly lower temperatures and masses for $t_{\mathrm{rel}}>0$.8. The $v \sin i$ range is rather narrow throughout the MS with a mean value of about $120 \mathrm{~km} \mathrm{~s}^{-1}$.

- There seems to exist a non-uniform distribution of effective temperatures for group members with a large proportion of objects (more than $70 \%$ ) cooler than $8000 \mathrm{~K}$.

- Objects with peculiar hydrogen-line profiles are preferentially found among later stages of stellar evolution.

- No correlation of age with elemental abundance or projected rotational velocity has been detected.

- A comparison of the stellar $\mathrm{Na}$ abundances with nearby IS lines of sight hints at an interaction between the $\lambda$ Bootis stars and the ISM.

- There is a single mechanism responsible for the observed phenomenon which produces moderate to strong underabundances working continuously from very early (10 Myr) to very late evolutionary stages $(2.5 \mathrm{Gyr})$. It produces the same absolute abundances throughout the MS lifetime for $2 \%$ of all luminosity class V objects with effective temperatures from $10500 \mathrm{~K}$ to $6500 \mathrm{~K}$.

- The current list of stars seem to define a very homogeneous group, validating the proposed membership criteria in the optical and UV region.

\section{Developed theories}

\subsection{Diffusion/mass-loss}

The diffusion/mass-loss theory was formulated by Michaud \& Charland (1986). They have modified the highly successful diffusion model responsible for the AmFm phenomenon to account for stellar mass-loss. AmFm stars are Population I nonmagnetic MS stars with underabundances of $\mathrm{Ca}$ and $\mathrm{Sc}$, but large overabundances of most heavier elements (up to a factor of 500). This abundance pattern is explained by the disappearance of the outer convection zone associated with the He-ionization because of the gravitional settling of He. Using Ti and Ca as examples, Michaud \& Charland (1986) showed that this model is capable of producing metal underabundances with the ad hoc assumption of a mass-loss rate of $10^{-13} M_{\odot} \mathrm{yr}^{-1}$. This high mass-loss rate impedes He settling enough to prevent the disappearance of the superficial He convection zone. Material originally located deep in the envelope then has time to be advected to the surface. Michaud \& Charland (1986) showed that the required underabundances materialize after $10^{9} \mathrm{yr}$ at the end of the MS lifetime for a normal A-type star. It was often quoted that this model only predicts underabundances of a factor five which is much too low compared to observations. But considering the poorly known parameters such as the diffusion coefficients, the scale heights and the boundaries of the convection zone, it might well be possible to obtain larger underabundances with this model. More of a problem is the rotational velocity and therefore meridional circulation. What manifests 
in a cut-off rotational velocity for AmFm stars $\left(\approx 90 \mathrm{~km} \mathrm{~s}^{-1}\right)$ also destroys the predicted $\lambda$ Bootis pattern. Charbonneau (1993) showed that even a moderate equatorial rotational velocity of $50 \mathrm{~km} \mathrm{~s}^{-1}$ prevents at any time during the MS evolution the appearance of the underabundance pattern because of mixing in the stellar atmosphere.

\subsection{Accretion/diffusion}

The accretion/diffusion model: Venn \& Lambert (1990) were the first who noticed the similarity between the abundance pattern of $\lambda$ Bootis stars and the depletion pattern of the interstellar medium (ISM) and suggested the accretion of interstellar or circumstellar gas to explain the $\lambda$ Bootis stars. In the ISM metals are underabundant because of their incorporation in dust grains or ice mantles around the dust grains. Waters et al. (1992) worked out a scenario, where the $\lambda$ Bootis star accretes metal-depleted gas from a surrounding disk. In this model, the dust grains are blown away by radiation pressure and coupling between dust and gas is negligible. Considering the spectral-type of $\lambda$ Bootis stars, the gas in the disk remains neutral and hence does not experience significant direct radiation pressure. The authors showed, that these conditions hold for mass accretion rates below $10^{-8} M_{\odot} \mathrm{yr}^{-1}$ assuming that the gas-to-dust mass ratio in the disk is 100 and that the disk consists of $0.1 \mu \mathrm{m}$ carbon grains. Turcotte \& Charbonneau (1993) calculated the abundance evolution in the outer layers of a $\lambda$ Bootis star assuming accretion rates between $10^{-15}$ and $10^{-12} M_{\odot} \mathrm{yr}^{-1}$. Solving the diffusion equation modified this time by an additional accretion term, they obtained the time evolution of the $\mathrm{Ca}$ and Ti abundance stratification with and without stellar rotation. From their calculations, a lower limit of $10^{-14} M_{\odot} \mathrm{yr}^{-1}$ is derived for the accretion/diffusion model to produce a typical $\lambda$ Bootis abundance pattern. Moreover their rotating models provide evidence that meridional circulation cannot destroy the established accretion pattern for rotational velocities smaller than $125 \mathrm{~km} \mathrm{~s}^{-1}$. Since diffusion wipes out any accretion pattern within $10^{6} \mathrm{yr}$ a large number of $\lambda$ Bootis stars should show observational evidence for the presence of circumstellar material. Turcotte (2002) revised this model recently applying new results on the depth of mixing in A-type stars. Richard et al. (2001) showed that the peculiar abundance pattern in Am stars (slow rotators) can only be reproduced by models in which the mixed layers extend much deeper than the superficial convection zone. For $\lambda$ Bootis stars, their study predicts a mixed zone of $\sim 10^{-6}$ times the stellar mass. Inserting this into the accretion/diffusion model increases the accretion rate, which is necessary for the accretion pattern to establish, to $10^{-11}$ to $10^{-12} \mathrm{M}_{\odot} \mathrm{yr}^{-1}$ depending on the exact mass of the mixed zone and the rotational velocity of the star. Andrievsky \& Paunzen (2000) studied the accretion from a circumstellar shell in more detail. They concluded that the gas and the dust decouple beyond the condensation radius of a $\lambda$ Bootis star. Assuming that the dust grains form at the condensation radius, grains will grow only to sizes less than $0.01 \mu \mathrm{m}$ before they are blown out of the shell. Moreover, the shell accretion can only cause a significant alteration of the photospheric abundances if the density in the shell is proportional to $r^{-2}$. Otherwise the shell contains too much material inside the condensation radius, which is not depleted due to grain formation. All the above discussed scenarios imply a constraint on the evolutionary status of the star, because the existence of a disk or a shell has to be explained in the context of stellar evolution. Circumstellar disks are thought to exist during the pre-main-sequence phase of stellar evolution, while a shell can either occur in a very early phase of pre-main-sequence evolution or after a stellar merger. Kamp \& Paunzen (2002) proposed only recently a slightly different accretion scenario for the $\lambda$ Bootis stars, namely the accretion from a diffuse interstellar cloud. This scenario works at any stage of stellar evolution as soon as the star passes a diffuse interstellar cloud. The interstellar dust grains are blown away 
by the stellar radiation pressure, while the depleted interstellar gas is accreted onto the star. Typical gas accretion rates are between $10^{-14}$ and $10^{-10} \mathrm{M}_{\odot} \mathrm{yr}^{-1}$ depending on the density of the diffuse cloud and the relative velocity between star and cloud. The hot limit for this model is due to strong stellar winds for stars with $T_{\text {eff }}>12000 \mathrm{~K}$ whereas the cool limit is defined by convection which prevents the accreted material to manifest at the stellar surface.

\subsection{Binary theories}

Binary theories: First, Andrievsky (1997) proposed that at least some $\lambda$ Bootis type stars can originate "by merging" as a result of the dynamical evolution of W UMa contact binary systems. These are close eclipsing binary stars with orbital periods less than one day. Spectral types of both components are almost always similar, mainly in the range between F0 and K0. Due to the angular momentum loss, both components of such a close system approach each other, and finally merge into a more massive but single star. The typical time for merging is poorly defined in the literature with values ranging from 100 - 200 Myr up to 500 Myr (Leonard \& Linnell 1992). The most essential point is that the merging occurs before both stars finish their Main Sequence lifetime. Since the masses of $\lambda$ Bootis stars can be found in the range between 1.5 and $2.6 M_{\odot}$ (Paunzen et al. 2002a), their progenitors could be W UMa close binary systems with masses of each component between 0.8 and $1.5 M_{\odot}$. In a nonconservative case Andrievsky (1997) proposes that some matter could be lost by the system during the merging phase and form a circumstellar shell. The above described scenario offers a simple observational test: the search for CNOprocessed material which should be mixed into the photospheres of $\lambda$ Bootis stars as a results of the merger process. But investigations show no abundance anomalies for $\mathrm{C}, \mathrm{N}$, $\mathrm{O}$ and $\mathrm{S}$ in many $\lambda$ Bootis stars. Andrievsky's suggestion is an attempt to bring into line the apparently evolved nature of some $\lambda$ Bootis type stars with the requirements of the accretion/diffusion model. It can be neither proved nor rejected, but clearly points to the model's limitations: slow selective accretion has a short time-scale, protostellar shells or disks would be swept out by a stellar wind soon after $(\approx 1 \mathrm{Myr})$ the ignition of core hydrogen burning and/or photoevaporated by nearby $\mathrm{O}$ or B-type stars. An interesting consequence, if some $\lambda$ Bootis type stars are binary star mergers, is they could have a common origin with the well-known blue stragglers. For a description of the latter see Stryker (1993). On the other hand Holweger et al. (1995) found through a detailed NLTE abundance analysis, that two blue stragglers in M67 do not show the typical $\lambda$ Bootis abundance pattern. Instead these two stars fit in the diffusion picture of normal A-type stars. Later Faraggiana \& Bonifacio (1999) and Gerbaldi et al. (2003) consider the metalweak appearance of the $\lambda$ Bootis spectra, and discuss the possibility that some if not most of the stars of this type are unresolved spectroscopic binaries. Thus, the observed weakness of the metallic lines could be an artefact. The single but composite spectrum of two quite normal (solar abundant) stars with different effective temperatures and gravities will have metal-weak character. The imitation would be even more "realistic" if the components have different rotational velocities. Speckle interferometry by Marchetti et al. (2001) and inspection of the isolated spectral features like the O I NIR-triplet at $\lambda 7770, \mathrm{NaD}$ lines and hydrogen line cores by Faraggiana et al. (2001) are used in an attempt to reveal the signs of binarity in the spectra of $\lambda$ Bootis type stars. Not surprisingly, only very few stars classified earlier as $\lambda$ Bootis with prominent shell-like characteristics prove to be binaries (HD 38545), or more complex systems (HD 111786). Undetected binarity gives a simple and attractive explanation of the peculiar hydrogen profiles which are typical for most of the $\lambda$ Bootis stars. But still it has to be proven that underabundances of up to -2 dex can be achieved by this model. 


\section{Acknowledgements}

This work benefitted from the Fonds zur Förderung der wissenschaftlichen Forschung, project (S7303-AST and P14984).

\section{References}

Abt, H.A., Morrell, N.I. 1995, ApJS 99, 135

Andrievsky, S.M. 1997, A\&̈A 321, 838

Andrievsky, S.M., Paunzen, E. 2000, MNRAS 313, 547

Bohlender, D.A., Gonzalez, J.F., Matthews, J.M. 1999, A\&\&A 350, 553

Charbonneau, P. 1993, ApJ 405, 720

Fajardo-Acosta, S.B., Stencel, R.E., Backman, D.E., Thakur, N. 1999, ApJ 520, 215

Faraggiana, R., Bonifacio, P. 1999, A $\mathscr{S} A$ 349, 521

Faraggiana, R., Gerbaldi, M., Böhm, C. 1990, A\& A 235, 311

Faraggiana, R., Gerbaldi, M., Bonifacio, P., Francois, P. 2001, A\&A 376, 586

Gerbaldi, M., Faraggiana, R., Lai, O. 2003, A\& $A$ 412, 447

Gray, R.O. 1988, $A J$ 95, 220

Gray, R.O., Corbally, C.J. 2002, AJ 124, 989

Heiter, U. 2002, A\& $A$ 381, 959

Holweger, H., Lemke, M., Rentzsch-Holm, I., Stürenburg, S. 1995, in: M. Busso, C.M. Raiteri and R. Gallino (eds.), Proceedings of the Symposium held at the National Laboratories of Gran Sasso, Assergi, L'Aquil, Italy, July 1994, AIP Conference Proceedings, vol. 327, p. 41

Kamp, I., Paunzen, E. 2002, MNRAS 335, L45

King, J.R. 1994, MNRAS 269, 209

Leonard, P.J.T., Linnell, A.P. 1992, AJ 103, 1928

Marchetti, E., Faraggiana, R., Bonifacio, P. 2001, A $\xi A$ 370, 524

Michaud, G., Charland, Y. 1986, ApJ 311, 326

Paunzen, E. 2001, A\&A 373, 633

Paunzen, E., Duffee, B., Heiter, U., Kuschnig, R., Weiss, W.W. 2001, A\&\&A 373, 625

Paunzen, E., Iliev, I.Kh., Kamp, I., Barzova, I.S. 2002a, MNRAS 336, 1030

Paunzen, E., Handler, G., Weiss, W.W., et al. 2002b, A\&3A 392, 515

Paunzen, E., Kamp, I., Weiss, W.W., Wiesemeyer, H. 2003, A\&\&A 404, 579

Richard, O., Michaud, G., Richer, J. 2001, ApJ 558, 377

Slettebak, A., Kuzma, T.J., Collins, G.W. 1980, ApJ 242, 171

Solano, E., Paunzen, E. 1998, A\& A 331, 633

Solano, E., Paunzen, E. 1999, A $\& A$ 348, 825

Stryker, L. L. 1993, PASP 105, 1081

Turcotte, S. 2002, ApJ 573, L129

Turcotte, S., Charbonneau, P. 1993, ApJ 413, 376

Venn, K.A., Lambert, D.L. 1990, ApJ 363, 234

Waters, L.B.F.M., Trams, N.R., Waelkens, C. 1992, A\&A 262, L37 